\title{
Volatile profiles and antioxidant activity of different cultivars of Camellia sinensis var. assamica grown in Thailand
}

\author{
${ }^{1}$ Khruengsai, S., ${ }^{1}$ Sripahco, T. and ${ }^{1,2,3, *}$ Pripdeevech, P. \\ ${ }^{1}$ School of Science, Mae Fah Luang University, Chiang Rai, 57100, Thailand \\ ${ }^{2}$ Center of Chemical Innovation for Sustainability (CIS), Mae Fah Luang University, Chiang Rai, 57100, \\ Thailand \\ ${ }^{3}$ Tea and Coffee Institute, Mae Fah Luang University, Chiang Rai, 57100, Thailand
}

\begin{abstract}
Article history:
Received: 13 October 2020

Received in revised form: 9

November 2020

Accepted: 1 January 2021

Available Online: 18 April

2021
\end{abstract}

Keywords:

Antioxidant,

Assam tea,

Camellia sinensis var.

assamica,

Catechin,

Phenolic compound,

Volatile compounds

\section{DOI:}

https://doi.org/10.26656/fr.2017.5(2).581

\begin{abstract}
Tea is considered as the most consumed drink in the world containing high antioxidant capacity. In this study, the volatile compounds, the phenolic content, catechins and caffeine including antioxidant activities of 22 Camellia sinensis var. assamica (Assam tea) cultivars were investigated. The volatile compounds were investigated by GC-MS. At least forty-five volatile compounds representing $94.99-99.65 \%$ of all cultivars were identified. Limonene, trans-linalool oxide, cis-linalool oxide, linalool, and furfural were detected as the major components among these cultivars. Varied ranges were found in all Assam tea cultivars for the contents of phenolics (113.45-245.55 mg gallic acid/g dry weight), total catechins (170.03-355.59 mg/g dry weight), caffeine $(0.92-3.40 \mathrm{mg} / \mathrm{g}$ dry weight), and antioxidant activities (1418.68-2728.46 $\mu \mathrm{mol}$ Trolox/g dry weight and 1448.98-2864.17 $\mu \mathrm{mol}$ Trolox/g dry weight for DPPH and ABTS assay, respectively). The antioxidant activity was correlated with phenolic compounds such as epigallocatechin gallate, epicatechin gallate, and catechin gallate. The specific differences among Assam tea cultivars are dependent on the tea cultivar and altitude which may play a significant role in breeding Assam tea cultivars in Thailand for providing its potential health benefits.
\end{abstract}

\section{Introduction}

Tea produced from Camellia sinensis leaves is the second most popular drink throughout the world next to water (Pripdeevech et al., 2017). Tea drinking has been reported to help to reduce the risk of cancer and heart diseases (Almajano et al., 2008). This benefit is the result of various types of bioactive compounds in tea such as polyphenols, caffeine, and terpenes accounting for its pharmaceutical and aroma properties (Kumazawa and Masuda, 2002). Polyphenol compounds in tea, mainly catechins, were detected up to $30-35 \%$ of the dry weight of tea leaves consisting of epicatechin, epicatechingallate, epigallocatechin, epigallocatechingallate, catechin, catechingallate, gallocatechin and gallocatechingallate (Pripdeevech and Machan, 2011). Studies have shown that these catechins are reported to possess antioxidant, anti-inflammatory, antibacterial and anti-allergic activities (Afzal et al., 2015). Caffeine has been reported to improve mood, attention, performance, alertness, the speed at which information is processed and reaction time (Astill et al., 2001). In addition, it was associated with reducing the risk of Parkinson's disease
(Li et al., 2012). Volatile aroma components in tea were particularly monoterpenes and sesquiterpenes with its derivatives achieving by fermentation processing methods (Pripdeevech and Machan, 2011).

The amount of the bioactive compounds in tea reflected its quality according to several parameters including cultivars, harvest season, age of the plant, climate, environmental and processing conditions as well as storage (Zeng et al., 2016). The tea cultivar is one of the most important factors affecting tea quality, and it also indicates types and relevant bioactive compound of tea. According to their genetics and cultivars, teas are classified into two main types including Chinese tea (Camellia sinensis var. sinensis) and Assam tea (Camellia sinensis var. assamica). Tea leaves of both types are processed to produce specific types of tea including green, oolong, and black tea.

In Thailand, tea is cultivated mainly in Chiang Rai and Chiang Mai provinces locating in the northern part of the country accounting for $93 \%$ of tea production. Commercially tea products in Thailand are obtained 
from both Assam and Chinese cultivar. About 30\% of tea products are commercialized in the domestic market, whereas $70 \%$ is exported (Theppakorn et al., 2014). In Thailand, Assam tea is cultivated in a larger planting area than Chinese tea (Theppakorn et al., 2014). It is mainly used to produce Miang or fermented tea (Theppakorn et al., 2012). It is indicated a lifestyle staple of people in northern Thailand especially hill tribes. It is used to welcome house guests or in northern style ceremonies and cultures (Phromrukachat et al., 2010). Although the potential of the various bioactive compound of Assam tea is similar to those found in Chinese tea, the price of Miang is lower than those of Chinese tea due to its astringent flavour from tannins and inappropriate taste of the ratio of sugar, fat, and chlorophylls (Reichart et al., 2005).

To our knowledge, there is less literature on the study of phytochemical profiles and the associated antioxidant activities of different Assam tea cultivars grown in the same conditions. It is of interest to know which tea cultivar could potentially be more beneficial in terms of antioxidant activity. Therefore, the objectives for this study were to determine the volatile profiles, antioxidant activities and total phenolic content in 22 Assam tea cultivars grown in the same conditions.

\section{Materials and methods}

\subsection{Plant material}

Shoots with a bud and two leaves from 22 cultivars of Assam tea (C. sinensis var. assamica) plants were harvested from the tea garden of the Tea and Coffee Institute, Mae Fah Luang University at BoonRod Rai, Chiang Rai province, Thailand in March 2019. The leaves were immediately processed at $220^{\circ} \mathrm{C}$ to promote inactivation of the endogenous enzymatic reaction prior to rolling for 30 mins using typical manufacturing approaches from BoonRod Rai. They were stored in the dry place at room temperature until analysis. Assam tea samples obtained from 22 cultivars are listed in Table 1.

\subsection{Chemicals}

HPLC-grade acetonitrile, methanol and trifluoroacetic acid were obtained from Merck (Darmstadt, Germany). Water was prepared using an ultrapure water system. The following standards were purchased from Sigma Chemical Co. (Thailand): gallic acid (GA), catechin (C), epicatechin (EC), epicatechin gallate (ECG), gallocatechin (GC), epigallocatechin gallate (EGCG), gallocatechin gallate (GCG), epigallocatechin (EGC), catechin gallate (CG), caffeine and Trolox. All solvents were of analytical grade, mixtures of $\mathrm{C}_{8}-\mathrm{C}_{17} \quad$ n-alkanes, 2,2-diphenyl-1-
Table 1. List of 22 Assam tea cultivars

\begin{tabular}{ccccc}
\hline No. & Province & Location & Altitude $(\mathrm{m})$ & Abbreviation \\
\hline 1 & Changrai & Maechan & 1058 & CR1 \\
2 & Changrai & Maechan & 853 & CR2 \\
3 & Changrai & Maung & 1012 & CR3 \\
4 & Changrai & Mae Suai & 563 & CR4 \\
5 & Changrai & Pan & 450 & CR5 \\
6 & Changrai & Thoeng & 420 & CR6 \\
7 & Changrai & Maefahluang & 1311 & CR7 \\
8 & Lampang & Maungpan & 1161 & LP1 \\
9 & Lampang & Ngaao & 800 & LP2 \\
10 & Nan & Muang & 737 & NN1 \\
11 & Nan & Pua & 1452 & NN2 \\
12 & Chiangmai & Omkoi & 855 & CM1 \\
13 & Chiangmai & Chiangdao & 1264 & CM2 \\
14 & Chiangmai & Maetaeng & 751 & CM3 \\
15 & Chiangmai & Chiangdao & 1253 & CM4 \\
16 & Chiangmai & Maetaeng & 700 & CM5 \\
17 & Chiangmai & Maetaeng & 712 & CM6 \\
18 & Chiangmai & Maetaeng & 716 & CM7 \\
19 & Chiangmai & Doisaket & 536 & CM8 \\
20 & Chiangmai & Doisaket & 521 & CM9 \\
21 & Chiangmai & Doisaket & 502 & CM10 \\
22 & Chiangmai & Doisaket & 548 & CM11 \\
\hline
\end{tabular}

picrylhydrazyl (DPPH) and 2,2-azinobis(3ethylbenzothiazoline-6-sulfonic acid) diammonium (ABTS) were purchased from Merck (Darmstadt, Germany).

\subsection{Identification of volatile compounds}

A $\quad 50 / 30 \quad \mu \mathrm{m} \quad$ divinylbenzene-carboxenpolydimethylsiloxane (DVB/CAR/PDMS) solid-phase microextraction (SPME) fibre purchased from Supelco (Bellefonte, PA, U.S.A.) was chosen to extract the volatile components from all Assam tea samples. The identification of volatile compounds in Assam tea samples was performed according to our previous research (Pripdeevech et al., 2017). For each extraction, the SPME fibre was preconditioned in the injection port of the Agilent 6890 gas chromatograph (Agilent Technologies, Inc., Palo Alto, CA, USA) at $220^{\circ} \mathrm{C}$ for 1 hr. Approximately, $100 \mathrm{~g}$ of each dried Assam tea sample were placed in a $250 \mathrm{~mL}$ headspace vial. The sample bottle was preheated at $60^{\circ} \mathrm{C}$ for 30 mins. The fibre was then exposed to the sample headspace for 30 mins, prior to thermal desorption of the constituents at $250^{\circ} \mathrm{C}$ into the split-less injection port of the gas chromatography-mass spectrometry (GC-MS) for 5 mins. SPME was performed in triplicate for each sample. The volatile components of all Thai Assam tea samples were analysed using a Hewlett Packard model HP6890 gas chromatograph (Agilent Technologies) equipped with an HP model 5973 mass-selective detector. An HP5MS (5\% phenylpolymethylsiloxane) capillary column 
$(30 \mathrm{~m} \times 0.25 \mathrm{~mm}$ i.d., film thickness $0.25 \mu \mathrm{m}$; Agilent Technologies) was used in this study. The oven temperature was set to $60^{\circ} \mathrm{C}$ and then increased to $220^{\circ} \mathrm{C}$ at a rate of $3^{\circ} \mathrm{C} / \mathrm{min}$. The injector and detector temperatures were set to $250^{\circ} \mathrm{C}$ and $280^{\circ} \mathrm{C}$, respectively. Purified helium $(99.9 \%$ ) was used as the carrier gas at a flow rate of $1 \mathrm{~mL} / \mathrm{min}$. Electron ionization (EI) mass spectra were collected at $70 \mathrm{eV}$ ionization voltages over the range of $\mathrm{m} / \mathrm{z} 29$ to 300 . The electron multiplier voltage was $1150 \mathrm{~V}$. The ion source and quadrupole temperatures were set to $230^{\circ} \mathrm{C}$ and $150^{\circ} \mathrm{C}$, respectively. Identification of volatile components was performed by comparison of their Kovát retention indices, relative to $\mathrm{C}_{8}-\mathrm{C}_{17} \mathrm{n}$-alkanes, and comparison of the mass spectra of individual components with reference mass spectra in the Wiley7N, NIST05 databases and Adams (2017). The relative contents of volatile compounds were calculated as peak areas to the peak area percentage

\subsection{Determination of total phenolic contents}

Total phenolic content of Assam tea infusions obtained from different cultivars was determined using the Folin-Ciocalteu reagent according to the method of Pripdeevech and Machan (2011) using gallic acid as standard. The tea solution $(0.2 \mathrm{~mL})$ was mixed with 1.0 $\mathrm{mL}$ of Folin-Ciocalteu reagent, $1.0 \mathrm{~mL}$ of an aqueous solution of $7 \% \mathrm{Na}_{2} \mathrm{CO}_{3}$ and $5.0 \mathrm{~mL}$ of distilled water, respectively. Then, the mixture was vortexed vigorously. The reaction mixtures were allowed to stand for $30 \mathrm{~min}$ before absorbance at $765 \mathrm{~nm}$ was measured. The same procedure was also applied to the standard solutions of gallic acid. The calibration equation for gallic acid was y $=0.00512 \mathrm{x}-0.00404\left(\mathrm{R}^{2}=0.999\right)$ where $\mathrm{x}$ and $\mathrm{y}$ are the absorbance and the concentration of gallic acid in $\mathrm{mg} /$ $\mathrm{mL}$, respectively. The total phenolic contents of all tea infusions are expressed as $\mathrm{mg}$ gallic acid $(\mathrm{GA}) / \mathrm{g}$ dried weight $(\mathrm{dw})$. The experiment was carried out in triplicate and the results are the mean values.

\subsection{Catechins and caffeine analysis}

Catechins and caffeine of Assam tea infusions obtained from different cultivars were determined according to the method of Theppakorn (2012). Each Assam tea infusion was prepared by adding $200 \mathrm{~mL}$ of boiling distilled water to $2 \mathrm{~g}$ of ground tea sample. Stirring using a magnetic stirrer was employed during the brewing process. After 10 mins, the tea infusion was filtered under vacuum and cooled to room temperature. Distilled water was added to tea solution until the final volume was $250 \mathrm{~mL}$. Tea solution was further filtered through a $0.22-\mu \mathrm{m}$ syringe filter membrane. The filtrate was stored at $-20^{\circ} \mathrm{C}$ for further high-performance liquid chromatography (HPLC) analysis. Analysis of catechins and caffeine was performed using of a Waters model
2695 HPLC system equipped with a model 2996 photodiode array detector. A Waters Platinum EPS $\mathrm{C}_{18}$ $(7.0 \mathrm{~mm}$ i.d. $\times 53 \mathrm{~mm}$ ) reversed phase column (Milford, MA). Catechins and caffeine were separated by elution with a flow rate of $2.0 \mathrm{~mL} / \mathrm{min}$ at $30^{\circ} \mathrm{C}$ using a binary mobile phase of $0.05 \%$ trifluoroacetic acid/acetonitrile $(87: 13 \mathrm{v} / \mathrm{v})$ in reservoir A and acetonitrile in reservoir B for a total chromatographic run time of 10 mins. Detection of catechins and caffeine was accomplished with a UV detection wavelength of $210 \mathrm{~nm}$. Calibrations plots for quantification were constructed from the injection of authentic standards of catechins and caffeine. Individual catechins and caffeine of each sample were reported as $\mathrm{mg}$ compound/g dw. The experiment was carried out in triplicate and the results are the mean values.

\subsection{Antioxidant activity}

Each Assam tea infusion was prepared by adding $200 \mathrm{~mL}$ of boiling distilled water to $2 \mathrm{~g}$ of ground tea sample. Stirring using a magnetic stirrer was employed during the brewing process. After $10 \mathrm{mins}$, the tea infusion was filtered under vacuum and cooled to room temperature. The antioxidant capacity by DPPH assay was evaluated according to the modified method (Insawang et al., 2019). The tea infusion and Trolox were diluted in methanol. A $0.05 \mathrm{~mL}$ of various infusions and Trolox was mixed with $1.95 \mathrm{~mL}$ of 0.2 mol/L DPPH solution. The mixture was shaken vigorously and kept in the dark at $27^{\circ} \mathrm{C}$ for 30 mins. The absorbance of the mixture was determined at $517 \mathrm{~nm}$ using a spectrophotometer. Methanol was used as a blank solution. The antioxidant activity by ABTS assay was also determined according to the modified method (Insawang et al., 2019). The ABTS radical cation was prepared by mixing $7 \mathrm{mM}$ ABTS solution with $2.45 \mathrm{mM}$ potassium persulfate and kept in the dark at $27^{\circ} \mathrm{C}$. For each concentration, $50 \mathrm{~mL}$ of the tea infusion was mixed with $150 \mathrm{~mL}$ of ABTS solution before shaking vigorously and kept in the dark at $27^{\circ} \mathrm{C}$ for 5 mins. The absorbance of the solution was determined at $734 \mathrm{~nm}$ using a spectrophotometer. The antioxidant activity of Assam tea cultivars was reported as $\mu$ mol Trolox/g dw from both assays. Each sample was tested for antioxidant activity in triplicate.

\section{Results and discussion}

Identified volatile compounds in different tea cultivars analysed by GC-MS and their amounts are summarized in Table 2. Forty-nine volatile compounds were identified. All similar cultivars contained a similar number of volatile components. Monoterpene compounds such as hotrienol, cis and trans-linalool 
oxide, linalool, limonene and furfural were found to be the major components among these samples. Assam tea samples obtained from Chiang Rai cultivars present high content of hotrienol, allo-ocimene, Z- $\beta$-ocimene, and E- $\beta$ -ocimene, $\delta$-elemene, and $\gamma$-muurolene. Some volatile compounds, jasmone, Z-jasmonyl acetate, and Z-methyl jasmonate were present in Assam tea cultivar obtained from high altitudes such as CM2 and CM4 whilst they were lower in the different cultivar amongst Assam tea samples. It was found that volatile components have been changed in term of quality and quantity according to the tea cultivar (Pripdeevech and Machan, 2011).

The phenolic contents of 22 Assam tea cultivars are shown in Figure 1. Among all cultivars analysed, NN2 had the highest phenolic content $(248.45 \pm 0.12 \mathrm{mg} \mathrm{GA} / \mathrm{g}$ dw). CR4 cultivar had the lowest phenolic content $(113.06 \pm 0.13 \mathrm{mg} \mathrm{GA} / \mathrm{g} \mathrm{dw})$. In this study, there was a 2.2-fold difference in phenolic content between the highest and lowest-ranked Assam tea cultivars. The phenolic compounds being a large group of secondary metabolites in natural products are evaluated to be responsible for the health benefits in cardiovascular protection and anticancer effects associating with their strong antioxidant activities (Zhou et al., 2016).

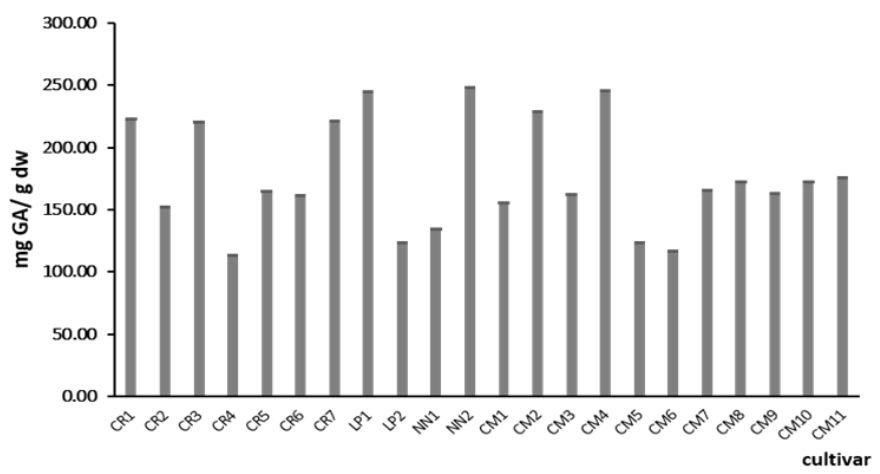

Figure 1. Total phenolic content of 22 Assam tea cultivars

The contents of represented catechins and caffeine were analysed by HPLC and the results are shown in Figure 2. All cultivars provided caffeine content ranging between $0.92-3.41 \mathrm{mg} / \mathrm{g} \mathrm{dw}$. Among all the tea cultivars analysed, CM5 and LP1 cultivars showed the lowest and highest caffeine content, respectively. Most tea cultivars had higher caffeine with more than $2.00 \mathrm{mg} / \mathrm{g} \mathrm{dw}$ except for CM5, CM6, and CM7 cultivar which was lower than $1.0 \mathrm{mg} / \mathrm{g} \mathrm{dw}$. In addition, CM4 had the highest total catechins content $(355.59 \pm 0.14 \mathrm{mg} / \mathrm{g} \mathrm{dw})$. CM10 had the lowest total catechins content $(170.03 \pm 0.78 \mathrm{mg} / \mathrm{g} \mathrm{dw})$. In evaluating tea cultivars for phenolic and catechin contents, a wide range has existed among these cultivars. The difference in content was mainly attributed to the different cultivars of Assam tea samples grown in the same conditions used in this study as reported by Fujimura et al. (2011). The amount of catechins in tea cultivar may result mainly in flavour and colour of tea and may be useful for the classification of tea cultivars and tea-making suitability (Wink, 2003). Catechins (EGCG, GC, EGC, and ECG) have been evaluated as the major phenols in almost all kinds of teas (Tong et al., 2019). EGCG has been reported as the richest catechins in tea and shown the strongest antioxidant activity in catechins, which is stronger than vitamins $\mathrm{C}$ and $\mathrm{E}$ (Zhao et al., 2014). It could possess several bioactivities such as anticancer activity by inhibiting cancer stem cells and modulating molecular actions associated with cancer cell proliferation, apoptosis, and immunity (Gan et al., 2018). Among all the Assam tea cultivars, CR7, CM4, CM2, and NN2 cultivars had high EGCG content (more than $134.00 \mathrm{mg} / \mathrm{g} \mathrm{dw}$ ). Other cultivars such as CR1, CR3, and LP1 showed moderate EGCG content ranging $113.23 \pm 0.29$ to $123.67 \pm 1.24 \mathrm{mg} / \mathrm{g} \mathrm{dw}$. Other cultivars had low EGCG content (lower than $100.00 \mathrm{mg} / \mathrm{g} \mathrm{dw}$ ). In this study, there was an approximately 2.3-fold difference in EGCG content between the highest and lowest-ranked cultivars. GC is another major catechin detected in all tested Assam tea cultivars. GC content ranged from 45.78 (CM10) to 128.77 (LP1) $\mathrm{mg} / \mathrm{g} \mathrm{dw}$. There were almost 2.8-fold differences in GC contents between CM10 and LP1. Wolfram et al. (2006) reported that a bitter and astringent taste in tea was attributed to GC content. Therefore, cultivars of Assam tea samples from CR7, CR3, CM4, CM2, NN2, and LP1 had a similar bitter taste due to the high content of GC

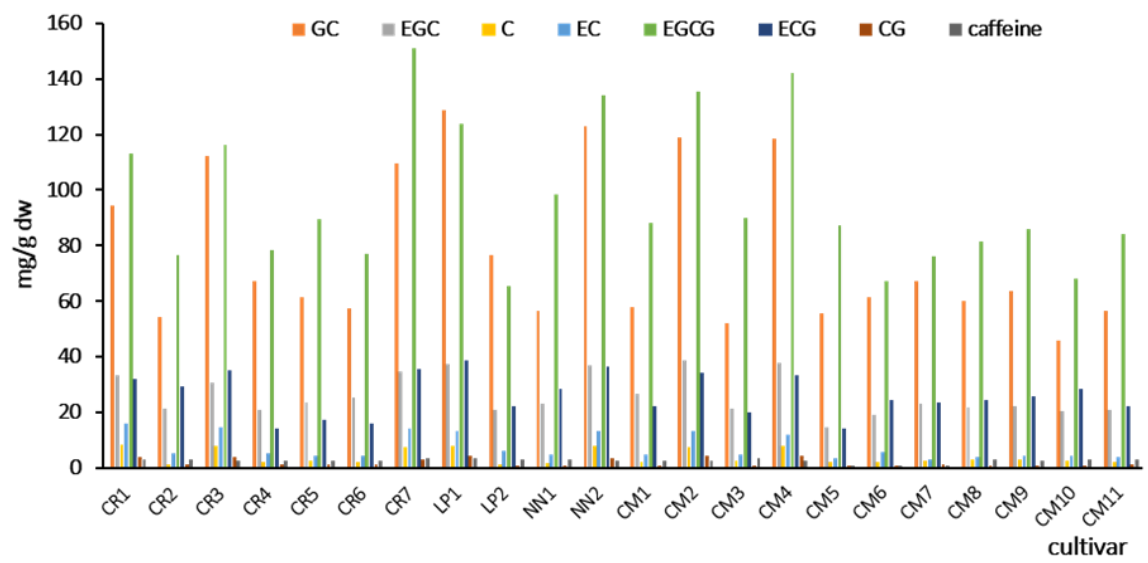

Figure 2. Catechins and caffeine content of 22 Assam tea cultivars 
$|\exists| \infty \infty \infty$ ฉ

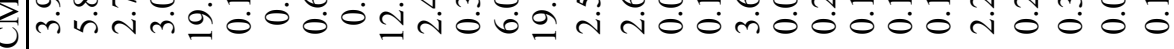

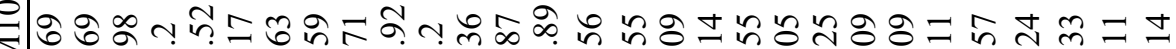

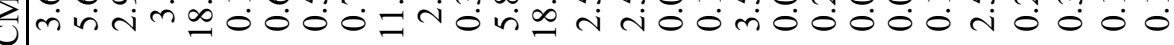

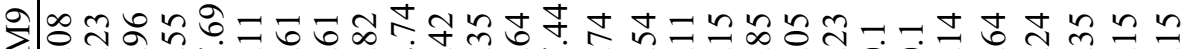
U min

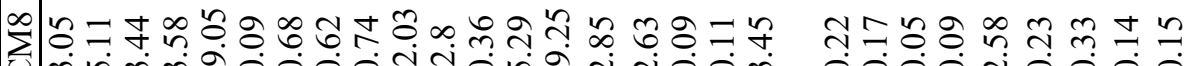
U minm

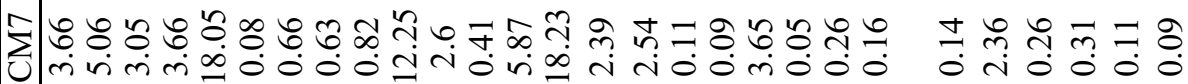

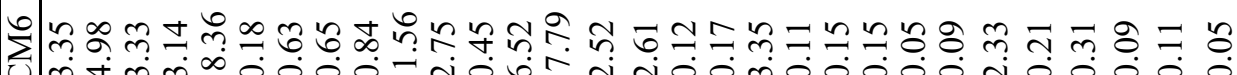
mnmann योن

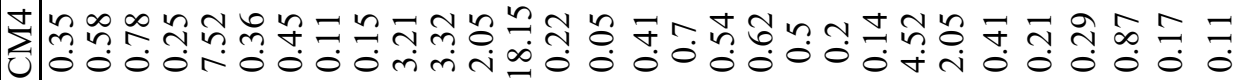

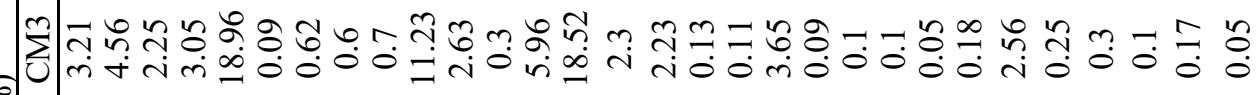

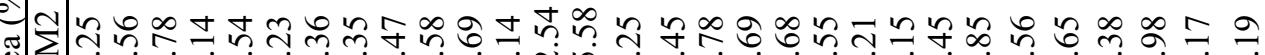
ل⿹

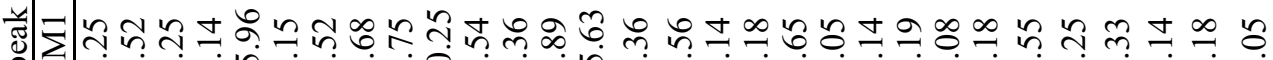

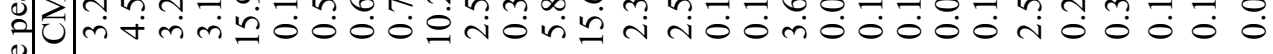

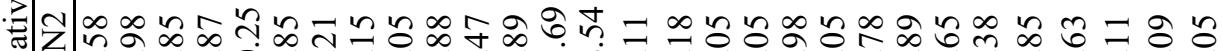
管

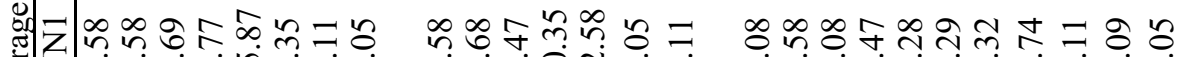

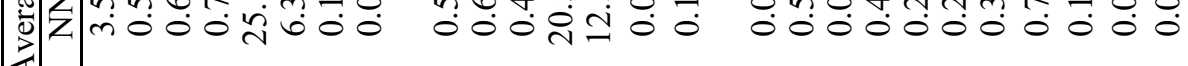

¿

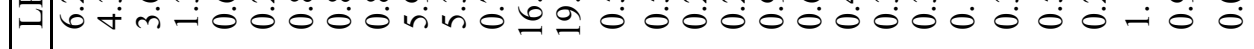
ప్ चn U

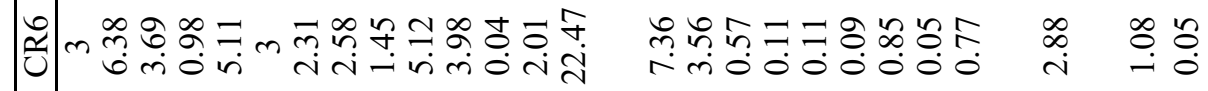
ڤิ

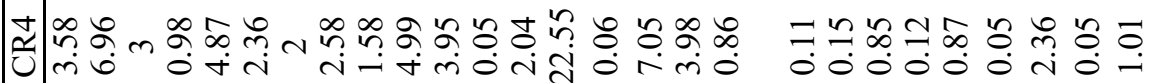

Uี

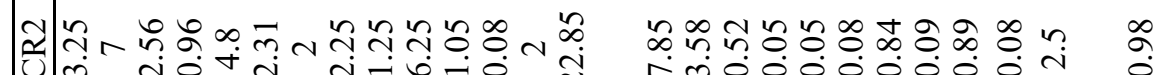

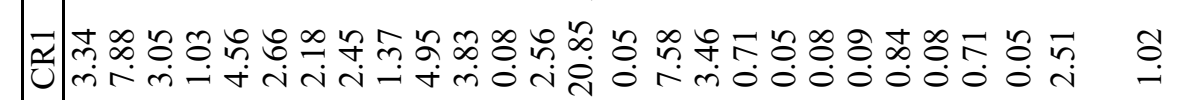

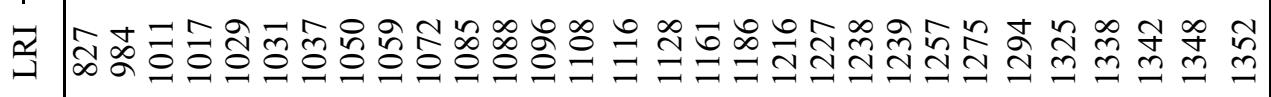




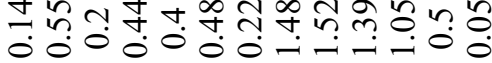

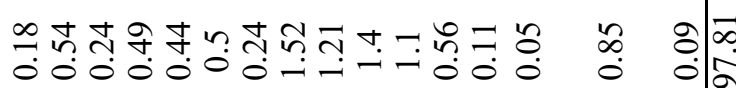

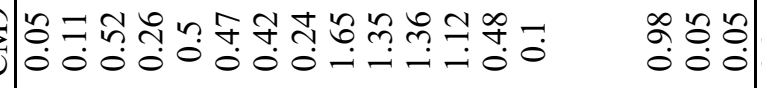

$\sum_{0}^{\infty}$ $00000000-1-i 00000000$

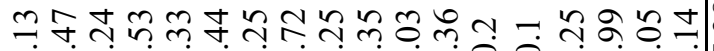
$0000000-1-10000000$

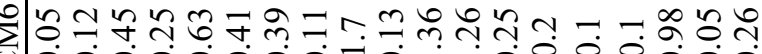
000000000000

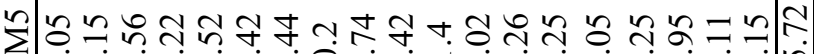

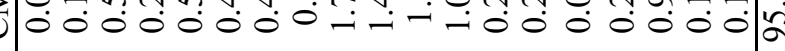

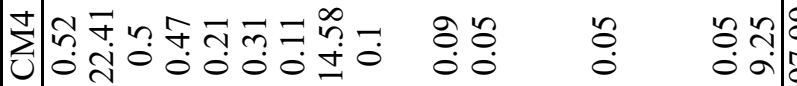

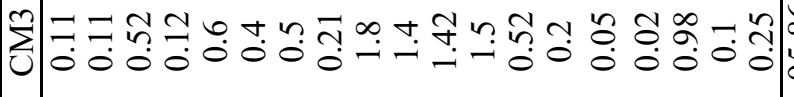

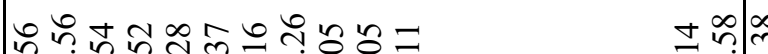
0 i 000000000

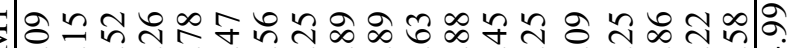
$0000000000-i-i 0-0.000000$

ఈ

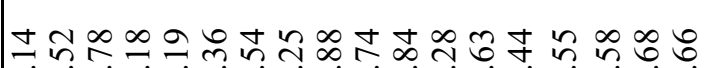
| $0000000000 \pi 000$ n 궝ำกำ

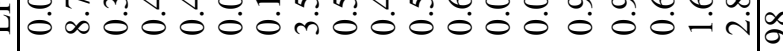

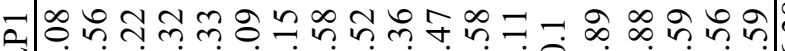

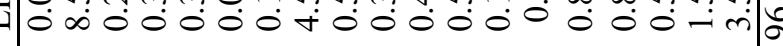

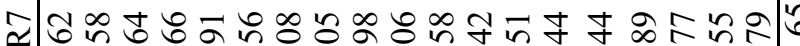

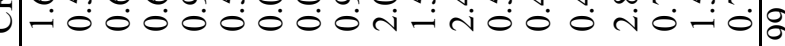

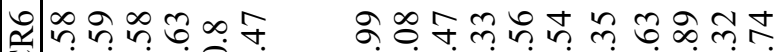

$\approx$ :

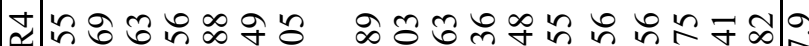

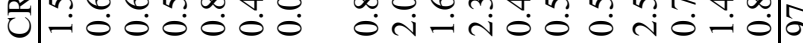

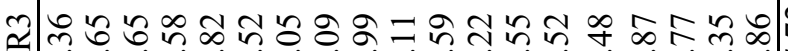
U - 0000000000

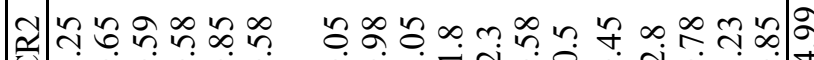

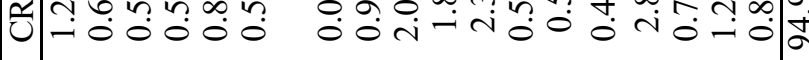

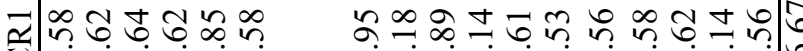

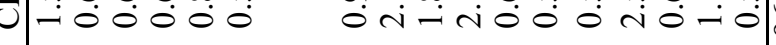

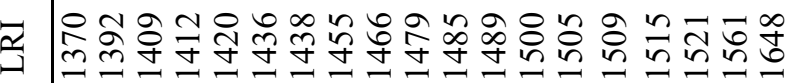


detected. Moreover, EGC and ECG contents were also detected as the major catechins in all cultivars ranging from $14.34-38.87 \mathrm{mg} / \mathrm{g} \mathrm{dw}$ and $14.23-38.45 \mathrm{mg} / \mathrm{g} \mathrm{dw}$, respectively. Among all the tested Assam tea cultivars, the phenolic and catechin contents of different Assam tea cultivars depended largely on the varietal difference (Zeng et al., 2016). Moreover, a previous study also demonstrated that the various contents of major catechin compounds including EGCG, GC, ECG and EGC in tea infusions were also related to the brewing time (Koch et al., 2017). Therefore, the optimization of brewing time may be further determined.

Due to the complexity of phytochemical compounds in tea, the antioxidant activities of tea were also evaluated. For this study, two assays, DPPH and ABTS were employed to determine comprehensively the antioxidant activities of tea infusions. The antioxidant activities of 22 Assam tea cultivars determined by both assays are expressed as $\mu \mathrm{mol}$ Trolox/g dw as shown in Figure 3. The antioxidant activity by DPPH assay (Figure 3A) ranged from $1234.45 \pm 0.17 \mu \mathrm{mol}$ Trolox/g $\mathrm{dw}$ (CR2) to $2728.46 \pm 0.24 \mu \mathrm{mol}$ Trolox/g dw (LP1). There was a 2.2-fold difference in DPPH values between the highest and lowest-ranked cultivars, CR2 and LP1. The ABTS values of 22 Assam tea cultivars are depicted in Figure 3B which was also expressed as $\mu \mathrm{mol}$ Trolox/g dw. Variation of ABTS values ranged from $1448.98 \pm 1.31 \mu \mathrm{mol}$ Trolox $/ \mathrm{g}$ dw (NN1) to $2864.97 \pm 1.12$ (NN2) $\mu$ mol Trolox/g dw. There was a 2.0-fold difference in ABTS value between the lowest and highest ranked cultivars, NN1 and NN2. Similar contents of antioxidant activity of 22 Assam tea cultivars were detected in both assays although some minor differences of antioxidant activity using DPPH and ABTS assays were detected. The values obtained from DPPH and ABTS of 22 tea infusions indicated that phenolic compounds were responsible for the strong antioxidant activities regarding reducing and free radicals scavenging abilities. However, it should be noted that the antioxidant activity of 22 Assam tea cultivars may be correlated to the presence of non-phenolic compounds such as polysaccharides. Sun et al. (2018). reported that four polysaccharides detected in green tea, with a molecular weight of $10.88,8.16,4.82$, and $2.31 \mathrm{kDa}$ possessed hydroxyl and ABTS radical scavenging activity.

From our results above, it can be noted that all Assam tea cultivars were evaluated to be the important tea having a good quality of antioxidant activity with an individual volatile profile. High antioxidant activity, total phenolic content and catechin compounds among these cultivars could be related to the low oxidation reaction of young tea leaves and bud due to inactivated enzymes during the sample preparation process (Senanayake, 2013). This observation is in agreement with the study of Zeng et al. (2016). The results suggested that tea cultivars had different antioxidant activity due to different phytochemicals. In addition, tea cultivars obtained from high altitudes above $1000 \mathrm{~m}$ such as CR1, CR3, CR7, LP1, NN2, CM2, and CM4 showed a higher amount of major catechin compounds than those obtained from other cultivars. Altitude maybe seemed to strongly outweigh their contribution due to the same tea production process.
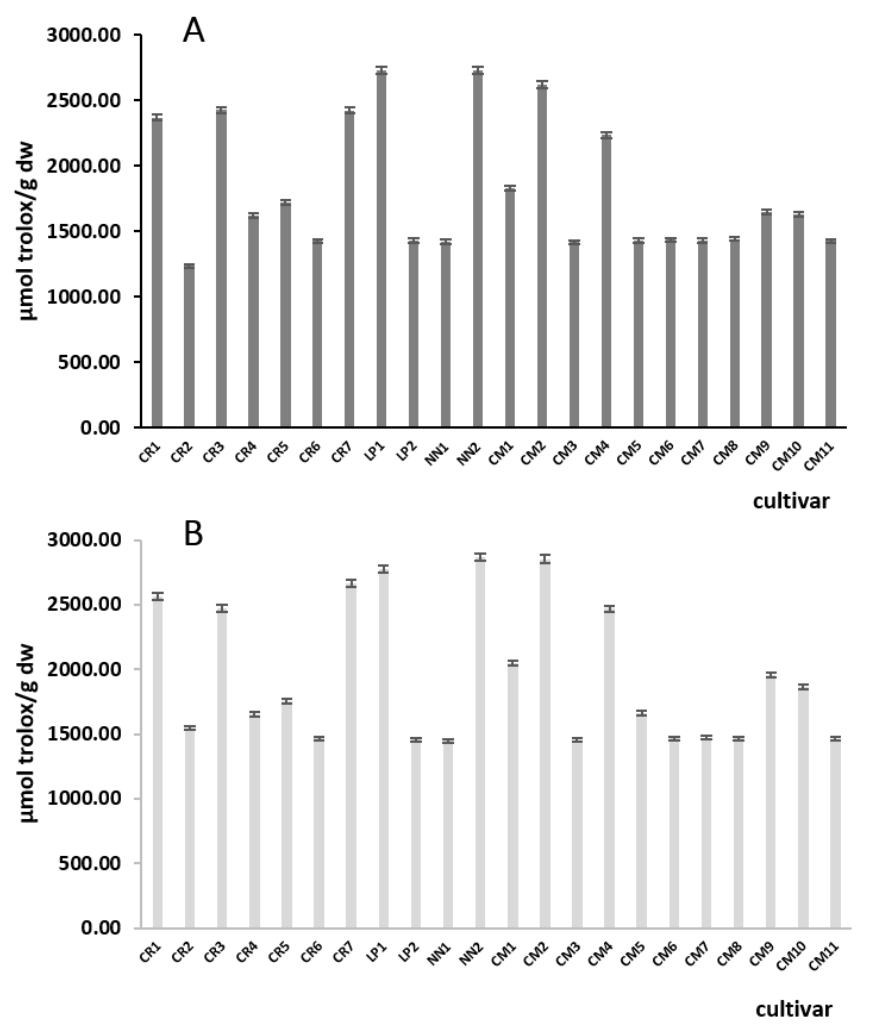

Figure 3. Antioxidant activity of 22 Assam tea cultivars by $\mathrm{DPPH}(\mathrm{A})$ and ABTS (B) assay

\section{Conclusion}

The present study comparatively determined the volatile profiles, total phenolic content, catechins, and antioxidant activity of Thai Assam tea cultivars grown in the same condition for the first time. The results suggested that 22 Assam tea infusions possessed specific volatile compounds characteristic, different antioxidant activities, phenolic content and catechin compounds varying in specific cultivars. The total antioxidant activities of Assam teas may be correlated with the major phenolic compounds among these cultivars such as EGCG, GC, ECG, and EGC. Tea cultivars obtained from high altitude show higher antioxidant activity, total phenolic and catechins content compared to other cultivars. Therefore, further research is needed to investigate the synergistic and individual effects of phytochemical compounds responsible for the antioxidant activity of Thai Assam tea. Knowledge of 
specific differences in the volatile profiles and antioxidant activities among Thai Assam tea cultivars is important data that may be used for breeding Assam tea cultivars in selecting for tea production in Thailand with various health benefits.

\section{Conflict of interest}

The authors declare no conflict of interest.

\section{Acknowledgments}

The authors wish to thank Mae Fah Luang University for financially support.

\section{References}

Adams, R.P. (2017). Identification of essential oil components by gas chromatography/mass spectrometry. Illinois, USA: Allured publishing Corporation: Carol Stream.

Afzal, M., Safer, A.M. and Menon, M. (2015). Green tea polyphenols and their potential role in health and disease. Inflammopharmacology, 23, 151-161. https://doi.org/.org/10.1007/s10787-015-0236-1

Almajano, M.P., Carbó, R., Jiménez, J.A.L. and Gordon, M.H. (2008). Antioxidant and antimicrobial activities of tea infusions. Food Chemistry, 108(1), 55-63.

https://doi.org/10.1016/ j.foodchem.2007.10.040

Astill, C., Birch, M.R., Dacombe, C., Humphrey, P.G. and Martin, P.T. (2001). Journal of Agricultural and Food Chemistry, 49, 5340-5347. https:// doi.org/10.1021/jf010759

Fujimura, Y., Kurihara, K., Ida, M., Kosaka, R., Miura, D., Wariishi, H., Maeda-Yamamoto, M., Nesumi, A., Saito, T., Kanda, T., Yamada, K. and Tachibana, H. (2011). Metabolomics-driven nutraceutical evaluation of diverse green tea cultivars. PLoS One, 6, e23426. https://doi.org/10.1371/ journal.pone. 0023426

Gan, R.Y., Li, H.B., Sui, Z.Q. and Corke, H. (2018). Absorption, metabolism, anti-cancer effect and molecular targets of epigallocatechin gallate (EGCG): An updated review. Critical Reviews in Food Science and Nutrition, 58(6), 924-941. https:// doi.org/10.1080/10408398.2016.1231168

Insawang, S., Pripdeevech, P., Tanapichatsakul, C., Khruengsai, S., Monggoot, S., Nakham, T., Artrod, A., D'Souza, P.E. and Panuwet, P. (2019). Essential oil compositions and antibacterial and antioxidant activities of five Lavandula stoechas cultivars grown in Thailand. Chemistry and Biodiversity, 16(10), e1900371. https://doi.org/10.1002/cbdv.201900371
Koch, W., Kukula-Koch, W. and Glowniak, K. (2017). Catechin composition and antioxidant activity of black teas in relation to brewing time. Journal of AOAC International, 100(6), 1694-1699. https:// doi.org/10.5740/jaoacint.17-0235

Kumazawa, K. and Masuda, H. (2002). Identification of potent odorants in different green tea varieties using flavor dilution technique. Journal of Agricultural and Food Chemistry, 50, 5660-5663. https:// doi.org/10.1021/jf020498j

Li, F.-J., Ji, H.-F. and Shen, L. (2012). A meta-analysis of tea drinking and risk of Parkinson's disease. The Scientific World Journal, 2012, 923464. https:// doi.org/10.1100/2012/923464

Phromrukachat, S., Tiengburanatum, N. and Meechui, J. (2010). Assessment of active ingredients in pickled tea. Asian Journal of Food and Agro-Industry, 3(3), 312-318.

Pripdeevech, P. and Machan, T. (2011). Fingerprint of volatile flavour constituents and antioxidant activities of teas from Thailand. Food Chemistry, 125(2), 797-802. https://doi.org/10.1016/ j.foodchem.2010.09.074

Pripdeevech, P., Rothwell, J., D'Souza, P.E. and Panuwet, P. (2017). Differentiation of volatile profiles of Thai Oolong tea No. 12 provenances by SPME-GC-MS combined with principal component analysis. International Journal of Food Properties, 20(Sup. 3), S2450-S2462. https:// doi.org/10.1080/10942912.2017.1374288

Reichart, P.A. and Philipsen, H.P. (2005). Betel and Miang: Vanishing Thai Habits. Bangkok, Thailand: White Lotus Books.

Senanayake, S. (2013). Green tea extract: Chemistry, antioxidant properties and food applications-A review. Journal of Functional Foods, 5(4), 15291541. https://doi.org/10.1016/j.jff.2013.08.011

Sun, X.Y., Wang, J.M., Ouyang, J.M. and Kuang, L. (2018). Antioxidant activities and repair effects on oxidatively damaged hk-2 cells of tea polysaccharides with different molecular weights. Oxidative Medicine and Cellular Longevity, 2018, 5297539. https://doi.org/10.1155/2018/5297539

Theppakorn, T. (2012). Tea (Camellia sinensis L.): Manufacturing and chemical compositions from fermentation. Burapha Science Journal, 17, 145-152. [In Thai].

Theppakorn, T., Luthfiyyah, A. and Ploysri, K. (2014). Comparison of the composition and antioxidant capacities of green teas produced from the Assam and the Chinese varieties cultivated in Thailand. Journal of Microbiology, Biotechnology and Food 
Sciences, 3(5), 364-370.

Theppakorn, T., Yamee, K. and Ekkapan, A. (2012). Green tea catechins and storage stability. $K K U$ Science Journal, 40(1), 1225-1235. [In Thai].

Tong, T., Liu, Y.J., Kang, J., Zhang, C.M. and Kang, S.G. (2019). Antioxidant activity and main chemical components of a novel fermented tea. Molecules, 24 (16), $2917 . \quad$ https://doi.org/10.3390/ molecules 24162917

Wink, M. (2003). Evolution of secondary metabolites from an ecological and molecular phylogenetic perspective. Phytochemistry, 64(1), 3-19. https:// doi.org/10.1016/S0031-9422(03)00300-5

Wolfram, S., Wang, Y. and Thielecke, F. (2006). Antiobesity effects of green tea: From bedside to bench. Molecular Nutrition Food Research, 50(2), 176-187. https://doi.org/10.1002/mnfr.200500102

Zeng, L., Luo, L., Li, H. and Liu, R. (2016). Phytochemical profiles and antioxidant activity of 27 cultivars of tea. International Journal of Food Sciences and Nutrition, 68(5), 525-537. https:// doi.org/10.1080/09637486.2016.1263834

Zhao, X., Wang, Q., Li, G., Chen, F., Qian, Y. and Wang, R. (2014). In vivo antioxidant, antimutagenic, anti-cancer and antiangiogenic effects of Chinese bowl tea. Journal of Functional Foods, 7, 590-598. https://doi.org/10.1016/j.jff.2013.12.026 